\title{
On the Solutions of Four Rational Difference Equations Associated to Tribonacci Numbers
}

\author{
İnci Okumuş, Yüksel Soykan
}
Zonguldak Bülent Ecevit University, Department of Mathematics,
Art and Science Faculty, 67100, Zonguldak, Turkey e-mail: inci_okumus_90@hotmail.com (corresponding author) yuksel_soykan@hotmail.com

\section{Abstract}

In this study, we investigate the form of solutions, stability character and asymptotic behavior of the following four rational difference equations

$$
\begin{aligned}
& x_{n+1}=\frac{1}{x_{n}\left(x_{n-1} \pm 1\right) \pm 1}, \\
& x_{n+1}=\frac{-1}{x_{n}\left(x_{n-1} \pm 1\right) \mp 1},
\end{aligned}
$$

such that their solutions are associated with Tribonacci numbers.

2010 Mathematics Subject Classification. 39A10, 39A30

Keywords: difference equations, solution, equilibrium point, tribonacci number, global asymptotic stability.

\section{Introduction}

Difference equations and systems of difference equations are great importance in the field of mathematics as well as in other sciences. The applications of the theory of difference equations appear as discrete mathematical models of many phenomena such as in biology, economics, ecology, control theory, physics, engineering, population dynamics and so forth. Recently, there has been a growing interest in the study of finding closed-form solutions of difference equations and systems of difference equations. Some of the forms of solutions of these equations are representable via well-known integer sequences such as Fibonacci numbers, Pell numbers, Lucas numbers and Padovan numbers. There are many papers on such these studies from several authors [1-17]. For example, in [14], Tollu et al. considered the following difference equations

$$
x_{n+1}=\frac{1}{1+x_{n}}, \quad y_{n+1}=\frac{1}{-1+y_{n}}, \quad n=0,1, \ldots,
$$

such that their solutions are associated with Fibonacci numbers. 
Then, in [17], Yazlik et al. studied the following rational difference equation systems

$$
x_{n+1}=\frac{x_{n-1} \pm 1}{y_{n} x_{n-1}}, \quad y_{n+1}=\frac{y_{n-1} \pm 1}{x_{n} y_{n-1}}, \quad n=0,1, \ldots,
$$

such that their solutions associated with Padovan numbers.

In [6], Halim concerned with the following systems of rational difference equations

$$
x_{n+1}=\frac{1}{1+y_{n}}, \quad y_{n+1}=\frac{1}{1+x_{n}}, \quad n=0,1, \ldots
$$

and

$$
x_{n+1}=\frac{1}{1-y_{n}}, \quad y_{n+1}=\frac{1}{1-x_{n}}, \quad n=0,1, \ldots,
$$

initial conditions are arbitrary nonzero real numbers.

In [7], Halim and Bayram investigated the solutions, stability character, and asymptotic behavior of the difference equation

$$
x_{n+1}=\frac{\alpha}{\beta+\gamma x_{n-k}}, n \in \mathbb{N}_{0},
$$

where the initial conditions $x_{-k}, x_{-k+1}, \ldots, x_{0}$ are nonzero real numbers, such that their solutions are associated to Horadam numbers, which are generalized Fibonacci numbers.

In [11], Öcalan and Duman considered the following nonlinear recursive difference equation

$$
x_{n+1}=\frac{x_{n-1}}{x_{n}}, n=0,1, \ldots,
$$

with any nonzero initial values $x_{-1}$ and $x_{0}$. Then, they extended their all results to solutions of the following nonlinear recursive equations

$$
x_{n+1}=\left(\frac{x_{n-1}}{x_{n}}\right)^{p}, p>0 \text { and } n=0,1, \ldots
$$

with any nonzero initial values $x_{-1}$ and $x_{0}$.

As far as we examine, there is no paper dealing with the following difference equations. Hence, in this study, we deal with the following difference equations

$$
\begin{array}{ll}
x_{n+1}=\frac{1}{x_{n}\left(x_{n-1}-1\right)-1}, & n=0,1, \ldots, \\
x_{n+1}=\frac{1}{x_{n}\left(x_{n-1}+1\right)+1}, & n=0,1, \ldots, \\
x_{n+1}=\frac{-1}{x_{n}\left(x_{n-1}-1\right)+1}, & n=0,1, \ldots, \\
x_{n+1}=\frac{-1}{x_{n}\left(x_{n-1}+1\right)-1}, & n=0,1, \ldots,
\end{array}
$$

such that their solutions are associated with Tribonacci numbers.

Our aim in this study is to investigate some relationships both between Tribonacci numbers and and solutions of above mentioned difference equations and between the Tribonacci constant and the equilibrium point of these difference equations. 
ON THE SOLUTIONS OF FOUR RATIONAL DIFFERENCE EQUATIONS ASSOCIATED TO TRIBONACCI NUMBERS

\section{Preliminaries}

2.1. Linearized stability. Let $I$ be some interval of real numbers and let $f: I^{k+1} \rightarrow I$ be a continuously differentiable function. A difference equation of order $(k+1)$ is an equation of the form

$$
x_{n+1}=f\left(x_{n}, x_{n-1}, \ldots, x_{n-k}\right), \quad n=0,1, \ldots
$$

A solution of Eq.(12) is a sequence $\left\{x_{n}\right\}_{n=-k}^{\infty}$ that satisfies Eq.(12) for all $n \geq-k$.

Definition 1. A solution of Eq.(12) that is constant for all $n \geq-k$ is called an equilibrium solution of Eq.(12). If

$$
x_{n}=\bar{x}, \text { for all } n \geq-k
$$

is an equilibrium solution of Eq.(12), then $\bar{x}$ is called an equilibrium point, or simply an equilibrium of Eq.(12)..

Definition 2 (Stability). Let $\bar{x}$ an equilibrium point of Eq.(12).

(a): An equilibrium point $\bar{x}$ of Eq.(12) is called locally stable if, for every $\varepsilon>0$; there exists $\delta>0$ such that if $\left\{x_{n}\right\}_{n=-k}^{\infty}$ is a solution of Eq.(12) with

$$
\left|x_{-k}-\bar{x}\right|+\left|x_{1-k}-\bar{x}\right|+\ldots+\left|x_{0}-\bar{x}\right|<\delta,
$$

then

$$
\left|x_{n}-\bar{x}\right|<\varepsilon, \text { for all } n \geq-k .
$$

(b): An equilibrium point $\bar{x}$ of Eq.(12) is called locally asymptotically stable if, it is locally stable, and if in addition there exists $\gamma>0$ such that if $\left\{x_{n}\right\}_{n=-k}^{\infty}$ is a solution of Eq.(12) with

$$
\left|x_{-k}-\bar{x}\right|+\left|x_{-k+1}-\bar{x}\right|+\ldots+\left|x_{0}-\bar{x}\right|<\gamma,
$$

then we have

$$
\lim _{n \rightarrow \infty} x_{n}=\bar{x}
$$

(c): An equilibrium point $\bar{x}$ of Eq.(12) is called a global attractor if, for every solution $\left\{x_{n}\right\}_{n=-k}^{\infty}$ of Eq.(12), we have

$$
\lim _{n \rightarrow \infty} x_{n}=\bar{x}
$$

(d): An equilibrium point $\bar{x}$ of Eq.(12) is called globally asymptotically stable if it is locally stable, and a global attractor.

(e): An equilibrium point $\bar{x}$ of Eq.(12) is called unstable if it is not locally stable.

Suppose that the function $f$ is continuously differentiable in some open neighborhood of an equilibrium point $\bar{x}$. Let

$$
q_{i}=\frac{\partial f}{\partial u_{i}}(\bar{x}, \bar{x}, \ldots, \bar{x}), \text { for } i=0,1, \ldots, k
$$

denote the partial derivative of $f\left(u_{0}, u_{1}, \ldots, u_{k}\right)$ with respect to $u_{i}$ evaluated at the equilibrium point $\bar{x}$ of Eq.(12). 
Definition 3. The equation

$$
y_{n+1}=q_{0} y_{n}+q_{1} y_{n-1}+\ldots+q_{k} y_{n-k}, n=0,1, \ldots
$$

is called the linearized equation of Eq.(12) about the equilibrium point $\bar{x}$, and the equation

$$
\lambda^{k+1}-q_{0} \lambda^{k}-\ldots-q_{k-1} \lambda-q_{k}=0
$$

is called the characteristic equation of Eq.(13) about $\bar{x}$.

Theorem 4 (The Linearized Stability Theorem). Assume that the function $f$ is a continuously differentiable function defined on some open neighborhood of an equilibrium point $\bar{x}$. Then the following statements are true:

(a): When all the roots of characteristic equation (14) have absolute value less than one, then the equilibrium point $\bar{x}$ of Eq.(12) is locally asymptotically stable.

(b): If at least one root of characteristic equation (14) has absolute value greater than one, then the equilibrium point $\bar{x}$ of Eq.(12) is unstable.

Moreover, the equilibrium point $\bar{x}$ of Eq.(12) is called hyperbolic if no root of characteristic equation (14) has absolute value equal to one. If there exists a root of characteristic equation (14) with absolute value equal to one, then the equilibrium $\bar{x}$ is called nonhyperbolic.

An equilibrium point $\bar{x}$ of Eq.(12) is called a repeller if all roots of characteristic equation (14) have absolute value greater than one.

An equilibrium point $\bar{x}$ of Eq.(12) is called a saddle if one of the roots of characteristic equation (14) is greater and another is less than one in absolute value.

2.2. Tribonacci numbers. Now, we give information about Tribonacci numbers that we afterwards need in the paper.

The Tribonacci sequence $\left\{T_{n}\right\}_{n=0}^{\infty}$ is defined by the third-order recurrence relations

$$
T_{n+3}=T_{n+2}+T_{n+1}+T_{n},
$$

with initial conditions $T_{0}=0, T_{1}=1, T_{2}=1$. Also, it can be extended the Tribonacci sequence backward (negative subscripts) as

$$
T_{-n}=T_{-n+3}-T_{-n+2}-T_{-n+1} .
$$

It can be clearly obtained that the characteristic equation of (15) has the form

$$
x^{3}-x^{2}-x-1=0
$$

such that the roots

$$
\begin{aligned}
& \alpha=\frac{1+\sqrt[3]{19+3 \sqrt{33}}+\sqrt[3]{19-3 \sqrt{33}}}{3} \\
& \beta=\frac{1+\omega \sqrt[3]{19+3 \sqrt{33}}+\omega^{2} \sqrt[3]{19-3 \sqrt{33}}}{3} \\
& \gamma=\frac{1+\omega^{2} \sqrt[3]{19+3 \sqrt{33}}+\omega \sqrt[3]{19-3 \sqrt{33}}}{3}
\end{aligned}
$$


where $\alpha$ is called Tribonacci constant and

$$
\omega=\frac{-1+i \sqrt{3}}{2}=\exp (2 \pi i / 3)
$$

is a primitive cube root of unity. Therefore, Tribonacci sequence can be expressed using Binet formula

$$
T_{n}=\frac{\alpha^{n+1}}{(\alpha-\beta)(\alpha-\gamma)}+\frac{\beta^{n+1}}{(\beta-\alpha)(\beta-\gamma)}+\frac{\gamma^{n+1}}{(\gamma-\alpha)(\gamma-\beta)}
$$

Furthermore, there exist the following limit

$$
\lim _{n \rightarrow \infty} \frac{T_{n+r}}{T_{n}}=\alpha^{r}
$$

where $r \in \mathbb{Z}$ and $T_{n}$ is the $n$th Tribonacci number.

\section{Main Results}

In this section, we present our main results considering above mentioned difference equations. Our aim is to investigate the general solutions in explicit form of difference equations and the asymptotic behavior of solutions of difference equations.

3.1. The Difference Equation (8). In this subsection, we consider the Eq.(8), that is,

$$
x_{n+1}=\frac{1}{x_{n}\left(x_{n-1}-1\right)-1}, \quad n=0,1, \ldots,
$$

and investigate the dynamical behavior of solutions of Eq.(8).

Theorem 5. Let $\left\{x_{n}\right\}_{n=-1}^{\infty}$ be a solution of Eq.(8). Then, for $n=0,1,2, \ldots$, the form of solutions $\left\{x_{n}\right\}_{n=-1}^{\infty}$ is given by

$$
x_{n}=\frac{T_{-n} x_{-1} x_{0}+\left(T_{-(n+1)}+T_{-(n+2)}\right) x_{0}+T_{-(n+1)}}{T_{-(n+1)} x_{-1} x_{0}+\left(T_{-n}-T_{-(n+1)}\right) x_{0}+T_{-(n+2)}},
$$

where $T_{n}$ is the nth Tribonacci number and the initial conditions $x_{-1}, x_{0} \in \mathbb{R}-F$, with $F$ is the forbidden set of Eq.(8) given by

$$
F=\bigcup_{n=-1}^{\infty}\left\{\left(x_{-1}, x_{0}\right): T_{-(n+1)} x_{-1} x_{0}+\left(T_{-n}-T_{-(n+1)}\right) x_{0}+T_{-(n+2)}=0\right\} .
$$

Proof. We will prove this theorem by induction on $k$. For $k=0$, from Eq.(8),

$$
x_{1}=\frac{1}{x_{0}\left(x_{-1}-1\right)-1}=\frac{1}{x_{-1} x_{0}-x_{0}-1}=\frac{T_{-1} x_{-1} x_{0}+\left(T_{-2}+T_{-3}\right) x_{0}+T_{-2}}{T_{-2} x_{-1} x_{0}+\left(T_{-1}-T_{-2}\right) x_{0}+T_{-3}} .
$$

Now, we assume that

$$
x_{k}=\frac{T_{-k} x_{-1} x_{0}+\left(T_{-(k+1)}+T_{-(k+2)}\right) x_{0}+T_{-(k+1)}}{T_{-(k+1)} x_{-1} x_{0}+\left(T_{-k}-T_{-(k+1)}\right) x_{0}+T_{-(k+2)}}
$$


is true for all $1 \leq n \leq k$. Hence, we have to prove that it is true for $k+1$. Taking into account (16) and (20), we have

$$
\begin{aligned}
& x_{k+1} \\
= & \frac{1}{x_{k}\left(x_{k-1}-1\right)-1} \\
= & \frac{1}{\left(\frac{T_{-k} x_{-1} x_{0}+\left(T_{-(k+1)}+T_{-(k+2)}\right) x_{0}+T_{-(k+1)}}{T_{-(k+1)} x_{-1} x_{0}+\left(T_{-k}-T_{-(k+1)}\right) x_{0}+T_{-(k+2)}}\right)\left(\frac{T_{-(k-1)} x_{-1} x_{0}+\left(T_{-k}+T_{-(k+1)}\right) x_{0}+T_{-k}}{T_{-k} x_{-1} x_{0}+\left(T_{-(k-1)}-T_{-k}\right) x_{0}+T_{-(k+1)}}-1\right)-1} \\
= & \frac{T_{-(k+1)} x_{-1} x_{0}+\left(T_{-k}-T_{-(k+1)}\right) x_{0}+T_{-(k+2)}}{\left(T_{-(k-1)}-T_{-k}-T_{-(k+1)}\right) x_{-1} x_{0}+\left(T_{-(k+1)}-T_{-(k+2)}\right) x_{0}+T_{-k}-T_{-(k+1)}-T_{-(k+2)}} \\
= & \frac{T_{-(k+1)} x_{-1} x_{0}+\left(T_{-(k+2)}+T_{-(k+3)}\right) x_{0}+T_{-(k+2)}}{T_{-(k+2)} x_{-1} x_{0}+\left(T_{-(k+1)}-T_{-(k+2)}\right) x_{0}+T_{-(k+3)}},
\end{aligned}
$$

which ends the induction and the proof.

TheOREM 6. Eq.(8) has unique positive equilibrium point $\bar{x}=\alpha$ and $\alpha$ is saddle point.

Proof. Equilibrium point of Eq.(8) satisfy the equation

$$
\bar{x}=\frac{1}{\bar{x}(\bar{x}-1)-1} \text {. }
$$

After simplification, we get the following cubic equation

$$
\bar{x}^{3}-\bar{x}^{2}-\bar{x}-1=0 .
$$

The cubic equation (21) is the characteristic equation of the recurrence relation of the Tribonacci numbers in (17) having the unique real root $\alpha$. Therefore, the unique positive equilibrium point of Eq.(8) is $\bar{x}=\alpha$.

Now, we indicate that the equilibrium point of Eq.(8) is saddle point.

Let $I$ be an interval of real numbers and

$$
f: I^{2} \rightarrow I
$$

be a continuous function defined by

$$
f(x, y)=\frac{1}{x(y-1)-1} .
$$

Therefore, it follows that

$$
\begin{aligned}
& \frac{\partial f(x, y)}{\partial x}=\frac{-(y-1)}{(x(y-1)-1)^{2}}, \\
& \frac{\partial f(x, y)}{\partial y}=\frac{-x}{(x(y-1)-1)^{2}} .
\end{aligned}
$$


ON THE SOLUTIONS OF FOUR RATIONAL DIFFERENCE EQUATIONS ASSOCIATED TO TRIBONACCI NUMBER\$ Then, from (21)

$$
\begin{aligned}
\frac{\partial f(\bar{x}, \bar{x})}{\partial x} & =\frac{-(\alpha-1)}{(\alpha(\alpha-1)-1)^{2}} \\
& =\frac{1-\alpha}{\left(\alpha^{2}-\alpha-1\right)^{2}} \\
& =\frac{1-\alpha}{\left(\frac{1}{\alpha}\right)^{2}} \\
& =\alpha^{2}-\alpha^{3} \\
\frac{\partial f(\bar{x}, \bar{x})}{\partial y} & =\frac{-(\alpha+1),}{(\alpha(\alpha-1)-1)^{2}} \\
& =\frac{-\alpha}{\left(\alpha^{2}-\alpha-1\right)^{2}} \\
& =\frac{-\alpha}{\left(\frac{1}{\alpha}\right)^{2}} \\
& =-\alpha^{3},
\end{aligned}
$$

and the linearized equation of Eq.(8) about $\bar{x}=\alpha$ is

$$
z_{n+1}=-(\alpha+1) z_{n}+\left(-\alpha^{3}\right) z_{n-1}
$$

or equivalently

$$
z_{n+1}+(\alpha+1) z_{n}+\alpha^{3} z_{n-1}=0
$$

Therefore, the corresponding characteristic polynomial is

$$
\lambda^{2}+(\alpha+1) \lambda+\alpha^{3}=0
$$

Then, from Theorem (4), it is clearly seen that

$$
\lambda_{1,2}=\frac{-(\alpha+1) \pm \sqrt{-4 \alpha^{3}+\alpha^{2}+2 \alpha+1}}{2}
$$

and numerically

$$
\begin{aligned}
& \left|\lambda_{1}\right|=\left|\frac{-(\alpha+1)+\sqrt{-4 \alpha^{3}+\alpha^{2}+2 \alpha+1}}{2}\right|=0,11228<1 \\
& \left|\lambda_{2}\right|=\left|\frac{-(\alpha+1)-\sqrt{-4 \alpha^{3}+\alpha^{2}+2 \alpha+1}}{2}\right|=1,4314>1
\end{aligned}
$$

So, the equilibrium point $\alpha$ is a saddle point. This completes the proof.

3.2. The Difference Equation (9). In this subsection, we study the Eq.(9), that is,

$$
x_{n+1}=\frac{1}{x_{n}\left(x_{n-1}+1\right)+1}, \quad n=0,1, \ldots,
$$

and examine the dynamical behavior of solutions of Eq.(9). 
TheOREM 7. Let $\left\{x_{n}\right\}_{n=-1}^{\infty}$ be a solution of Eq.(9). Then, for $n=0,1,2, \ldots$, the form of solutions $\left\{x_{n}\right\}_{n=-1}^{\infty}$ is given by

$$
x_{n}=\frac{T_{n-1} x_{-1} x_{0}+\left(T_{n+1}-T_{n}\right) x_{0}+T_{n}}{T_{n} x_{-1} x_{0}+\left(T_{n-1}+T_{n}\right) x_{0}+T_{n+1}},
$$

where $T_{n}$ is the nth Tribonacci number and the initial conditions $x_{-1}, x_{0} \in \mathbb{R}-F$, with $F$ is the forbidden set of Eq.(9) given by

$$
F=\bigcup_{n=-1}^{\infty}\left\{\left(x_{-1}, x_{0}\right): T_{n} x_{-1} x_{0}+\left(T_{n-1}+T_{n}\right) x_{0}+T_{n+1}=0\right\} .
$$

Proof. (First proof) Now, we give the proof with an analytic approach. We make the substitution

$$
x_{n}=\frac{t_{n-1}}{t_{n}}
$$

in Eq.(9) to get the linear difference equation. Then, we have

$$
t_{n+1}=t_{n}+t_{n-1}+t_{n-2} \text {. }
$$

By using same operations in Theorem 2.1. in [??] such that $a=b=c=1$, we obtain the initial values of three sequences are defined

$$
\begin{aligned}
a_{n} & =a a_{n-1}+b a_{n-2}+c a_{n-3} \\
b_{n} & =a_{n+1}-a a_{n} \\
c_{n} & =c a_{n-1}
\end{aligned}
$$

such that

$$
\begin{aligned}
& a_{0}=1, \quad a_{-1}=0, \quad a_{-2}=0, \\
& b_{0}=0, \quad b_{-1}=1, \quad b_{-2}=0 \text {, } \\
& c_{0}=0, \quad c_{-1}=0, \quad c_{-2}=1 \text {. }
\end{aligned}
$$

Next, we get

$$
t_{n}=a_{n} t_{0}+\left(a_{n+1}-a a_{n}\right) t_{-1}+c a_{n_{-1}} t_{-2} \text {. }
$$

So $a=b=c=1$ and from (23), we obtain

or equivalently

$$
x_{n}=\frac{a_{n-2} x_{-1} x_{0}+\left(a_{n}-a_{n-1}\right) x_{0}+a_{n-1}}{a_{n-1} x_{-1} x_{0}+\left(a_{n+1}-a_{n}\right) x_{0}+a_{n}}
$$

$$
x_{n}=\frac{a_{n-2} x_{-1} x_{0}+\left(a_{n}-a_{n-1}\right) x_{0}+a_{n-1}}{a_{n-1} x_{-1} x_{0}+\left(a_{n-1}+a_{n-2}\right) x_{0}+a_{n}} .
$$

From initial values (24) and definitions of sequences $a_{n}$ and $T_{n}$, we have

$$
a_{n}=T_{n+1},
$$

with the backward shifted initial values of the sequence $a_{n}$. Hence, we obtain

$$
x_{n}=\frac{T_{n-1} x_{-1} x_{0}+\left(T_{n+1}-T_{n}\right) x_{0}+T_{n}}{T_{n} x_{-1} x_{0}+\left(T_{n-1}+T_{n}\right) x_{0}+T_{n+1}} .
$$

So, the proof is complete.

Proof. (Second proof) We will prove this theorem by induction on $k$. For $k=0$, from Eq.(9),

$$
x_{1}=\frac{1}{x_{0}\left(x_{-1}+1\right)+1}=\frac{1}{x_{-1} x_{0}+x_{0}+1}=\frac{T_{0} x_{-1} x_{0}+\left(T_{2}-T_{1}\right) x_{0}+T_{1}}{T_{1} x_{-1} x_{0}+\left(T_{0}+T_{1}\right) x_{0}+T_{2}} .
$$


ON THE SOLUTIONS OF FOUR RATIONAL DIFFERENCE EQUATIONS ASSOCIATED TO TRIBONACCI NUMBERg

Now, we assume that

$$
x_{k}=\frac{T_{k-1} x_{-1} x_{0}+\left(T_{k+1}-T_{k}\right) x_{0}+T_{k}}{T_{k} x_{-1} x_{0}+\left(T_{k-1}+T_{k}\right) x_{0}+T_{k+1}},
$$

is true for all $1 \leq n \leq k$. Hence, we have to prove that it is true for $k+1$. Taking into account (15) and (25), we have

$$
\begin{aligned}
x_{k+1} & =\frac{1}{x_{k}\left(x_{k-1}+1\right)+1} \\
& =\frac{1}{\left(\frac{T_{k-1} x_{-1} x_{0}+\left(T_{k+1}-T_{k}\right) x_{0}+T_{k}}{T_{k} x_{-1} x_{0}+\left(T_{k-1}+T_{k}\right) x_{0}+T_{k+1}}\right)\left(\frac{T_{k-2} x_{-1} x_{0}+\left(T_{k}-T_{k-1}\right) x_{0}+T_{k-1}}{T_{k-1} x_{-1} x_{0}+\left(T_{k-2}+T_{k-1}\right) x_{0}+T_{k}}+1\right)+1} \\
& =\frac{T_{k} x_{-1} x_{0}+\left(T_{k-1}+T_{k}\right) x_{0}+T_{k+1}}{\left(T_{k-2}+T_{k-1}+T_{k}\right) x_{-1} x_{0}+\left(T_{k}+T_{k+1}\right) x_{0}+T_{k-1}+T_{k}+T_{k+1}} \\
& =\frac{T_{k} x_{-1} x_{0}+\left(T_{k+2}-T_{k+1}\right) x_{0}+T_{k+1}}{T_{k+1} x_{-1} x_{0}+\left(T_{k}+T_{k+1}\right) x_{0}+T_{k+2}},
\end{aligned}
$$

which ends the induction and the proof.

Proof. (Third proof) Consider Eq.(9) by taking $n=0,1,2, \ldots$ as follows:

$$
\begin{array}{ccc}
n=0 & \Rightarrow & x_{1}=\frac{1}{x_{-1} x_{0}+x_{0}+1}, \\
n=1 & \Rightarrow & x_{2}=\frac{x_{-1} x_{0}+x_{0}+1}{x_{-1} x_{0}+2 x_{0}+2}, \\
n=2 & \Rightarrow & x_{3}=\frac{x_{-1} x_{0}+2 x_{0}+2}{2 x_{-1} x_{0}+3 x_{0}+4}, \\
n=3 & \Rightarrow & x_{4}=\frac{2 x_{-1} x_{0}+3 x_{0}+4}{4 x_{-1} x_{0}+6 x_{0}+7}, \\
n=4 & \Rightarrow & x_{5}=\frac{4 x_{-1} x_{0}+6 x_{0}+7}{7 x_{-1} x_{0}+11 x_{0}+13}, \\
n=5 & \Rightarrow & x_{6}=\frac{7 x_{-1} x_{0}+11 x_{0}+13}{13 x_{-1} x_{0}+20 x_{0}+24}, \\
& \ldots
\end{array}
$$

If we keep on this process and also regard (15), then the solution in (22) directly follows from a simple induction.

THEOREM 8. Eq.(9) has unique positive equilibrium point $\bar{x}=$ a and a is locally asymptotically stable.

Proof. Equilibrium point of Eq.(9) is the real roots of the equation

$$
\bar{x}=\frac{1}{\bar{x}(\bar{x}+1)+1} .
$$

After simplification, we get the following cubic equation

$$
\bar{x}^{3}+\bar{x}^{2}+\bar{x}-1=0 .
$$

Then, the roots of the cubic equation (26) are given by

$$
\begin{aligned}
& a=\frac{-1+\sqrt[3]{3 \sqrt{33}+17}-\sqrt[3]{3 \sqrt{33}-17}}{3} \\
& b=\frac{-1+\omega \sqrt[3]{3 \sqrt{33}+17}-\omega^{2} \sqrt[3]{3 \sqrt{33}-17}}{3} \\
& c=\frac{-1+\omega^{2} \sqrt[3]{3 \sqrt{33}+17}-\omega \sqrt[3]{3 \sqrt{33}-17}}{3}
\end{aligned}
$$

where

$$
\omega=\frac{-1+i \sqrt{3}}{2}=\exp (2 \pi i / 3)
$$


is a primitive cube root of unity. So, the root $a$ is only real number. Therefore, the unique positive equilibrium point of Eq.(9) is $\bar{x}=a$.

Now, we show that the equilibrium point of Eq.(9) is locally asymptotically stable.

Let $I$ be an interval of real numbers and consider the function

$$
f: I^{2} \rightarrow I
$$

defined by

$$
f(x, y)=\frac{1}{x(y+1)+1} .
$$

The linearized equation of Eq.(9) about the equilibrium point $\bar{x}=a$ is

$$
z_{n+1}=p z_{n}+q z_{n-1}
$$

where

$$
\begin{aligned}
p & =\frac{\partial f(\bar{x}, \bar{x})}{\partial x}=\frac{-(a+1)}{(a(a+1)+1)^{2}}=a-1, \\
q & =\frac{\partial f(\bar{x}, \bar{x})}{\partial y}=\frac{-a}{(a(a+1)+1)^{2}}=-a^{3}
\end{aligned}
$$

and the corresponding characteristic equation is

$$
\lambda^{2}+(1-a) \lambda+a^{3}=0
$$

Therefore, from Theorem (4), it is easily seen that

$$
\lambda_{1,2}=\frac{a-1 \pm \sqrt{-4 a^{3}+a^{2}-2 a+1}}{2}
$$

and numerically

$$
\left|\lambda_{1}\right|=\left|\lambda_{2}\right|=0,40089<1
$$

This completes the proof.

THeOREM 9. The equilibrium point of Eq.(9) is globally asymptotically stable.

Proof. Let $\left\{x_{n}\right\}_{n>-1}$ be a solution of Eq.(9). By Theorem (8), we need only to prove that the equilibrium point $a$ is global attractor, that is

$$
\lim _{n \rightarrow \infty} x_{n}=a \text {. }
$$

From Theorem (7), (17) and (18), it follows that

$$
\begin{aligned}
\lim _{n \rightarrow \infty} x_{n} & =\lim _{n \rightarrow \infty} \frac{T_{n-1} x_{-1} x_{0}+\left(T_{n+1}-T_{n}\right) x_{0}+T_{n}}{T_{n} x_{-1} x_{0}+\left(T_{n-1}+T_{n}\right) x_{0}+T_{n+1}} \\
& =\lim _{n \rightarrow \infty} \frac{T_{n-1}\left(x_{-1} x_{0}+\left(\frac{T_{n+1}}{T_{n-1}}-\frac{T_{n}}{T_{n-1}}\right) x_{0}+\frac{T_{n}}{T_{n-1}}\right)}{T_{n}\left(x_{-1} x_{0}+\left(\frac{T_{n-1}}{T_{n}}+1\right) x_{0}+\frac{T_{n+1}}{T_{n}}\right)} \\
& =\left(\frac{x_{-1} x_{0}+\left(\alpha^{2}-\alpha\right) x_{0}+\alpha}{x_{-1} x_{0}+\left(\frac{1}{\alpha}+1\right) x_{0}+\alpha}\right) \lim _{n \rightarrow \infty} \frac{T_{n-1}}{T_{n}} \\
& =\lim _{n \rightarrow \infty} \frac{T_{n-1}}{T_{n}} \\
& =\frac{1}{\alpha} \\
& =a .
\end{aligned}
$$


ON THE SOLUTIONS OF FOUR RATIONAL DIFFERENCE EQUATIONS ASSOCIATED TO TRIBONACCI NUMBER\$ The proof is complete.

3.3. The Difference Equation (10). In this subsection, we take into account the Eq.(10), that is,

$$
x_{n+1}=\frac{-1}{x_{n}\left(x_{n-1}-1\right)+1}, \quad n=0,1, \ldots,
$$

and analyze the dynamical behavior of solutions of Eq.(10).

Theorem 10. Let $\left\{x_{n}\right\}_{n=-1}^{\infty}$ be a solution of Eq.(10). Then, for $n=0,1,2, \ldots$, the form of solutions $\left\{x_{n}\right\}_{n=-1}^{\infty}$ is given by

$$
x_{n}=\frac{-\left(T_{n-1} x_{-1} x_{0}+\left(T_{n}-T_{n+1}\right) x_{0}+T_{n}\right)}{T_{n} x_{-1} x_{0}-\left(T_{n-1}+T_{n}\right) x_{0}+T_{n+1}},
$$

where $T_{n}$ is the nth Tribonacci number and the initial conditions $x_{-1}, x_{0} \in \mathbb{R}-F$, with $F$ is the forbidden set of Eq.(10) given by

$$
F=\bigcup_{n=-1}^{\infty}\left\{\left(x_{-1}, x_{0}\right): T_{n} x_{-1} x_{0}-\left(T_{n-1}+T_{n}\right) x_{0}+T_{n+1}=0\right\} .
$$

Proof. (First proof) We will prove this theorem by induction on $k$. For $k=0$, from Eq.(10),

$$
x_{1}=\frac{-1}{x_{0}\left(x_{-1}-1\right)+1}=\frac{-1}{x_{-1} x_{0}-x_{0}+1}=\frac{-\left(T_{0} x_{-1} x_{0}+\left(T_{1}-T_{2}\right) x_{0}+T_{1}\right)}{T_{1} x_{-1} x_{0}-\left(T_{0}+T_{1}\right) x_{0}+T_{2}} .
$$

Now, we assume that

$$
x_{k}=\frac{-\left(T_{k-1} x_{-1} x_{0}+\left(T_{k}-T_{k+1}\right) x_{0}+T_{k}\right)}{T_{k} x_{-1} x_{0}-\left(T_{k-1}+T_{k}\right) x_{0}+T_{k+1}}
$$

is true for all $1 \leq n \leq k$. Hence, we have to prove that it is true for $k+1$. Taking into account (15) and (28), we have

$$
\begin{aligned}
x_{k+1} & =\frac{-1}{x_{k}\left(x_{k-1}-1\right)+1} \\
& =\frac{-1}{\left(\frac{-\left(T_{k-1} x_{-1} x_{0}+\left(T_{k}-T_{k+1}\right) x_{0}+T_{k}\right)}{T_{k} x_{-1} x_{0}-\left(T_{k-1}+T_{k}\right) x_{0}+T_{k+1}}\right)\left(\frac{-\left(T_{k-2} x_{-1} x_{0}+\left(T_{k-1}-T_{k}\right) x_{0}+T_{k-1}\right)}{T_{k-1} x_{-1} x_{0}-\left(T_{k-2}+T_{k-1}\right) x_{0}+T_{k}}-1\right)+1} \\
& =\frac{-\left(T_{k} x_{-1} x_{0}-\left(T_{k-1}+T_{k}\right) x_{0}+T_{k+1}\right)}{\left(T_{k-2}+T_{k-1}+T_{k}\right) x_{-1} x_{0}-\left(T_{k}+T_{k+1}\right) x_{0}+T_{k-1}+T_{k}+T_{k+1}} \\
& =\frac{-\left(T_{k} x_{-1} x_{0}+\left(T_{k+1}-T_{k+2}\right) x_{0}+T_{k+1}\right)}{T_{k+1} x_{-1} x_{0}-\left(T_{k}+T_{k+1}\right) x_{0}+T_{k+2}}
\end{aligned}
$$

which ends the induction and the proof.

Proof. (Second proof) Consider Eq.(10) by taking $n=0,1,2, \ldots$ as follows:

$$
\begin{array}{cccc}
n=0 & \Rightarrow & x_{1}=\frac{-1}{x_{-1} x_{0}-x_{0}+1}, \\
n=1 & \Rightarrow & x_{2}=\frac{-\left(x_{-1} x_{0}-x_{0}+1\right)}{x_{-1} x_{0}-2 x_{0}+2}, \\
n=2 & \Rightarrow & x_{3}=\frac{-\left(x_{-1} x_{0}-2 x_{0}+2\right)}{2 x_{-1} x_{0}-3 x_{0}+4}, \\
n=3 & \Rightarrow & x_{4}=\frac{-\left(2 x_{-1} x_{0}-3 x_{0}+4\right)}{4 x_{-1} x_{0}-6 x_{0}+7} \\
n=4 & \Rightarrow & x_{5}=\frac{-\left(4 x_{-1} x_{0}-6 x_{0}+7\right)}{7 x_{-1} x_{0}-11 x_{0}+13} \\
n=5 & \Rightarrow & x_{6}=\frac{-\left(7 x_{-1} x_{0}-11 x_{0}+13\right)}{13 x_{-1} x_{0}-20 x_{0}+24} \\
& \ldots &
\end{array}
$$


If we keep on this process and also regard (15), then the solution in (27) directly follows from a simple induction.

Theorem 11. Eq.(10) has unique negative equilibrium point $\bar{x}=d$ and $d$ is locally asymptotically stable.

Proof. Equilibrium point of Eq.(10) is the real roots of the equation

$$
\bar{x}=\frac{-1}{\bar{x}(\bar{x}-1)+1} .
$$

After simplification, we get the following cubic equation

$$
\bar{x}^{3}-\bar{x}^{2}+\bar{x}+1=0 .
$$

Then, the roots of the cubic equation (29) are given by

$$
\begin{aligned}
& d=\frac{1+\sqrt[3]{3 \sqrt{33}-17}-\sqrt[3]{3 \sqrt{33}+17}}{3} \\
& e=\frac{1+\omega \sqrt[3]{3 \sqrt{33}-17}-\omega^{2} \sqrt[3]{3 \sqrt{33}+17}}{3} \\
& f=\frac{1+\omega^{2} \sqrt[3]{3 \sqrt{33}-17}-\omega \sqrt[3]{3 \sqrt{33}+17}}{3}
\end{aligned}
$$

where

$$
\omega=\frac{-1+i \sqrt{3}}{2}=\exp (2 \pi i / 3)
$$

is a primitive cube root of unity. So, the root $d$ is only real number. Therefore, the unique negative equilibrium point of Eq.(10) is $\bar{x}=d$.

Now, we show that the unique negative equilibrium point of Eq.(10) is locally asymptotically stable.

Let $I=(0, \infty)$ and consider the function

$$
f: I^{2} \rightarrow I
$$

defined by

$$
f(x, y)=\frac{-1}{x(y-1)+1} .
$$

The linearized equation of Eq.(10) about the equilibrium point $\bar{x}=d$ is

$$
z_{n+1}=p z_{n}+q z_{n-1}
$$

where, from (29),

$$
\begin{aligned}
& p=\frac{\partial f(\bar{x}, \bar{x})}{\partial x}=\frac{d-1}{(d(d-1)+1)^{2}}=\frac{d-1}{\left(d^{2}-d+1\right)^{2}}=\frac{d-1}{\left(-\frac{1}{d}\right)^{2}}=-(d+1), \\
& q=\frac{\partial f(\bar{x}, \bar{x})}{\partial y}=\frac{d}{(d(d-1)+1)^{2}}=\frac{d}{\left(d^{2}-d+1\right)^{2}}=\frac{d}{\left(-\frac{1}{d}\right)^{2}}=d^{3}
\end{aligned}
$$

and the corresponding characteristic equation is

$$
\lambda^{2}+(d+1) \lambda-d^{3}=0 .
$$

Therefore, from Theorem (4), it is easily seen that

$$
\lambda_{1,2}=\frac{-(d+1) \pm \sqrt{4 d^{3}+d^{2}+2 d+1}}{2}
$$


ON THE SOLUTIONS OF FOUR RATIONAL DIFFERENCE EQUATIONS ASSOCIATED TO TRIBONACCI NUMBERS and numerically

$$
\left|\lambda_{1}\right|=\left|\lambda_{2}\right|=0,40089<1
$$

So, this completes the proof.

THEOREM 12. The equilibrium point of Eq.(10) is globally asymptotically stable.

Proof. Let $\left\{x_{n}\right\}_{n \geq-1}$ be a solution of Eq.(10). By Theorem (11), we need only to prove that the equilibrium point $d$ is global attractor, that is

$$
\lim _{n \rightarrow \infty} x_{n}=d
$$

From Theorem (10), (17) and (18), it follows that

$$
\begin{aligned}
\lim _{n \rightarrow \infty} x_{n} & =\lim _{n \rightarrow \infty} \frac{-\left(T_{n-1} x_{-1} x_{0}+\left(T_{n}-T_{n+1}\right) x_{0}+T_{n}\right)}{T_{n} x_{-1} x_{0}-\left(T_{n-1}+T_{n}\right) x_{0}+T_{n+1}} \\
& =\lim _{n \rightarrow \infty} \frac{-\left(T_{n-1}\left(x_{-1} x_{0}+\left(\frac{T_{n}}{T_{n-1}}-\frac{T_{n+1}}{T_{n-1}}\right) x_{0}+\frac{T_{n}}{T_{n-1}}\right)\right)}{T_{n}\left(x_{-1} x_{0}-\left(\frac{T_{n-1}}{T_{n}}+1\right) x_{0}+\frac{T_{n+1}}{T_{n}}\right)} \\
& =\left(\frac{x_{-1} x_{0}+\left(\alpha-\alpha^{2}\right) x_{0}+\alpha}{x_{-1} x_{0}-\left(\frac{1}{\alpha}+1\right) x_{0}+\alpha}\right) \lim _{n \rightarrow \infty} \frac{-T_{n-1}}{T_{n}} \\
& =\lim _{n \rightarrow \infty} \frac{-T_{n-1}}{T_{n}} \\
& =-\frac{1}{\alpha} \\
& =d .
\end{aligned}
$$

The proof is complete.

3.4. The Difference Equation (11). In this subsection, we take into account the Eq.(11), that is,

$$
x_{n+1}=\frac{-1}{x_{n}\left(x_{n-1}+1\right)-1}, \quad n=0,1, \ldots,
$$

and analyze the dynamical behavior of solutions of Eq.(11).

TheOREM 13. Let $\left\{x_{n}\right\}_{n=-1}^{\infty}$ be a solution of Eq.(11). Then, for $n=0,1,2, \ldots$, the form of solutions $\left\{x_{n}\right\}_{n=0-1}^{\infty}$ is given by

$$
x_{n}=\frac{-\left(T_{-n} x_{-1} x_{0}-\left(T_{-(n+1)}+T_{-(n+2)}\right) x_{0}+T_{-(n+1)}\right)}{T_{-(n+1)} x_{-1} x_{0}+\left(T_{-(n+1)}-T_{-n}\right) x_{0}+T_{-(n+2)}},
$$

where $T_{n}$ is the $n$th Tribonacci number and the initial conditions $x_{-1}, x_{0} \in \mathbb{R}-F$, with $F$ is the forbidden set of Eq.(11) given by

$$
F=\bigcup_{n=-1}^{\infty}\left\{\left(x_{-1}, x_{0}\right): T_{-(n+1)} x_{-1} x_{0}+\left(T_{-(n+1)}-T_{-n}\right) x_{0}+T_{-(n+2)}=0\right\} .
$$

Proof. We will prove this theorem by induction on $k$. For $k=0$, from Eq.(11), $x_{1}=\frac{-1}{x_{0}\left(x_{-1}+1\right)-1}=\frac{-1}{x_{-1} x_{0}+x_{0}-1}=\frac{-\left(T_{-1} x_{-1} x_{0}\left(T_{-2}+T_{-3}\right) x_{0}+T_{-2}\right)}{T_{-2} x_{-1} x_{0}+\left(T_{-2}-T_{-1}\right) x_{0}+T_{-3}}$. 
Now, we assume that

$$
x_{k}=\frac{-\left(T_{-k} x_{-1} x_{0}-\left(T_{-(k+1)}+T_{-(k+2)}\right) x_{0}+T_{-(k+1)}\right)}{T_{-(k+1)} x_{-1} x_{0}+\left(T_{-(k+1)}-T_{-k}\right) x_{0}+T_{-(k+2)}}
$$

is true for all $1 \leq n \leq k$. Hence, we have to prove that it is true for $k+1$. Taking into account (16) and (31), we have

$$
\begin{aligned}
& x_{k+1} \\
= & \frac{-1}{x_{k}\left(x_{k-1}+1\right)-1} \\
= & \frac{-1}{\left(\frac{-\left(T_{-k} x_{-1} x_{0}-\left(T_{-(k+1)}+T_{-(k+2)}\right) x_{0}+T_{-(k+1)}\right)}{T_{-(k+1)} x_{-1} x_{0}+\left(T_{-(k+1)}-T_{-k}\right) x_{0}+T_{-(k+2)}}\right)\left(\frac{-\left(T_{-(k-1)} x_{-1} x_{0}-\left(T_{-k}+T_{-(k+1)}\right) x_{0}+T_{-k}\right)}{T_{-k} x_{-1} x_{0}+\left(T_{-k}-T_{-(k-1)}\right) x_{0}+T_{-(k+1)}}+1\right)-1} \\
= & \frac{-\left(T_{-(k+1)} x_{-1} x_{0}-\left(T_{-(k+2)}+T_{-(k+3)}\right) x_{0}+T_{-(k+2)}\right)}{\left(T_{-(k-1)}-T_{-k}-T_{-(k+1)}\right) x_{-1} x_{0}+\left(T_{-(k+2)}-T_{-(k+1)}\right) x_{0}+T_{-k}-T_{-(k+1)}-T_{-(k+2)}} \\
= & \frac{-\left(T_{-(k+1)} x_{-1} x_{0}-\left(T_{-(k+2)}+T_{-(k+3)}\right) x_{0}+T_{-(k+2)}\right)}{T_{-(k+2)} x_{-1} x_{0}+\left(T_{-(k+2)}-T_{-(k+1)}\right) x_{0}+T_{-(k+3)}},
\end{aligned}
$$

which ends the induction and the proof.

Theorem 14. Eq.(11) has unique negative equilibrium point $\bar{x}=a$ and $a$ unstable.

Proof. Equilibrium point of Eq.(11) satisfy the equation

$$
\bar{x}=\frac{-1}{\bar{x}(\bar{x}+1)-1} .
$$

After simplification, we get the following cubic equation

$$
\bar{x}^{3}+\bar{x}^{2}-\bar{x}+1=0 .
$$

Then, the roots of the cubic equation (32) are given by

where

$$
\begin{aligned}
& g=\frac{-1-\sqrt[3]{19+3 \sqrt{33}}-\sqrt[3]{19-3 \sqrt{33}}}{3} \\
& h=\frac{-1-\omega \sqrt[3]{19+3 \sqrt{33}}-\omega^{2} \sqrt[3]{19-3 \sqrt{33}}}{3} \\
& k=\frac{-1-\omega^{2} \sqrt[3]{19+3 \sqrt{33}}-\omega \sqrt[3]{19-3 \sqrt{33}}}{3}
\end{aligned}
$$

$$
\omega=\frac{-1+i \sqrt{3}}{2}=\exp (2 \pi i / 3)
$$

is a primitive cube root of unity. So, the root $g$ is only real number. Therefore, the unique negative equilibrium point of Eq.(11) is $\bar{x}=g$.

Now, we indicate that the negativeequilibrium point of Eq.(11) is unstable.

Let $I$ be an interval of real numbers and

$$
f: I^{2} \rightarrow I
$$

be a continuous function defined by

$$
f(x, y)=\frac{-1}{x(y+1)-1} .
$$


Therefore, it follows that

$$
\begin{aligned}
\frac{\partial f(x, y)}{\partial x} & =\frac{y+1}{(x(y+1)-1)^{2}} \\
\frac{\partial f(x, y)}{\partial y} & =\frac{x}{(x(y+1)-1)^{2}} .
\end{aligned}
$$

Then, from (32)

$$
\begin{aligned}
\frac{\partial f(\bar{x}, \bar{x})}{\partial x} & =\frac{g+1}{(g(g+1)-1)^{2}} \\
& =\frac{g+1}{\left(g^{2}+g-1\right)^{2}} \\
& =\frac{g+1}{\left(-\frac{1}{g}\right)^{2}} \\
& =g^{3}+g^{2} \\
\frac{\partial f(\bar{x}, \bar{x})}{\partial y} & =\frac{g-1,}{(g(g+1)-1)^{2}} \\
& =\frac{g}{\left(g^{2}+g-1\right)^{2}} \\
& =\frac{g}{\left(-\frac{1}{g}\right)^{2}} \\
& =g^{3},
\end{aligned}
$$

and the linearized equation of Eq.(11) about $\bar{x}=g$ is

$$
z_{n+1}=(g-1) z_{n}+g^{3} z_{n-1}
$$

or equivalently

$$
z_{n+1}-(g-1) z_{n}-g^{3} z_{n-1}=0 .
$$

Therefore, the corresponding characteristic polynomial is

$$
\lambda^{2}-(g-1) \lambda-g^{3}=0 .
$$

Then, from Theorem (4), it is clearly seen that

$$
\lambda_{1,2}=\frac{(g-1) \pm \sqrt{4 g^{3}+g^{2}-2 g+1}}{2}
$$

and numerically

$$
\left|\lambda_{1}\right|=\left|\lambda_{2}\right|=2,4944>1 \text {. }
$$

So, the equilibrium point $g$ is unstable. This completes the proof.

\section{References}

[1] Alotaibi, AM, Noorani, MSM, El-Moneam, MA, On the Solutions of a System of Third-Order Rational Difference Equations. Discrete Dynamics in Nature and Society, Article ID 1743540, 11 pages (2018)

[2] Akrour, Y, Touafek, N, Halim, Y, On a System of Difference Equations of Second Order Solved in a Closed Form. arXiv:1904.04476v1, [math.DS] (2019) 
[3] Bacani, JB, Rabago, JFT, On Two Nonlinear Difference Equations. Dynamics of Continuous, Discrete and Impulsive Systems, (Serias A) to appear (2015)

[4] El-Dessoky, On the dynamics of higher order difference equations $x_{n+1}=a x_{n}+$ $\frac{\alpha x_{n} x_{n-l}}{\beta x_{n}+\gamma x_{n-k}}$. J. Computational Analysis and Applications, 22(7): 1309-1322 (2017)

[5] El-Dessoky, MM, Elabbasy, EM, Asiri A, Dynamics and Solutions of a Fifth-Order Nonlinear Difference Equations. Discrete Dynamics in Nature and Society, Article ID 9129354, 21 pages (2018)

[6] Halim, Y, Global Character of Systems of Rational Difference Equations. Electronic Journal of Mathematical Analysis and Applications, 3(1): 204-214 (2015)

[7] Halim, Y, Bayram, M, On the solutions of a higher-order difference equation in terms of generalized Fibonacci sequences. Mathematical Methods in the Applied Sciences, 39: 2974$2982(2016)$

[8] Halim, Y, A System of Difference Equations with Solutions Associated to Fibonacci Numbers. International Journal of Difference Equations, 11(1): 65-77 (2016)

[9] Halim, Y, Rabago, JFT, On Some Solvable Systems of Difference Equations with Solutions Associated to Fibonacci Numbers. Electronic Journal of Mathematical Analysis and Applications, 5(1): 166-178 (2017)

[10] Halim, Y, Rabago, JFT, On the Solutions of a Second-Order Difference Equation in terms of Generalized Padovan Sequences. Mathematica Slovaca, 68(3): 625-638 (2018)

[11] Öcalan, Ö, Duman, O, On Solutions of the Recursive Equations $x_{n+1}=x_{n-1}^{p} / x_{n}^{p}(p>0)$ via Fibonacci-Type Sequences. Electronic Journal of Mathematical Analysis and Applications, 7(1): 102-115 (2019)

[12] ??Rabago, JFT, On the Closed-Form Solution of a Nonlinear Difference Equation and Another Proof to Sroysang's Conjecture. arXiv:1604.06659v1 [math.NT] (2016)

[13] Stevic, S, Iricanin, B, Kosmala, W, Smarda, Z, Representation of solutions of a solvable nonlinear difference equation of second order. Electronic Journal of Qualitative Theory of Differential Equations, 95: 1-18 (2018)

[14] Tollu, DT, Yazlik, Y, Taskara, N, On the solutions of two special types of Riccati difference equation via Fibonacci numbers. Advances in Difference Equations, 2013:174 (2013)

[15] Tollu, DT, Yazlik, Y, Taskara, N, The Solutions of Four Riccati Difference Equations Associated with Fibonacci numbers. Balkan Journal of Mathematics, 2: 163-172 (2014)

[16] Tollu, DT, Yazlik, Y, Taskara, N, On fourteen solvable systems of difference equations. Applied Mathematics and Computation, 233: 310-319 (2014)

[17] Yazlik, Y, Tollu, DT, Taskara, N, On the Solutions of Difference Equation Systems with Padovan Numbers. Applied Mathematics, 4:15-20 (2013) 